\title{
Responses of atmospheric circulation to sea surface temperature anomalies in the South China Sea
}

\author{
M. P. Zhou and G. H. Wang
}

State Key Laboratory of Satellite Ocean Environment Dynamics, Second Institute of Oceanography, State Oceanic Administration, 310012 Hangzhou, China

Correspondence to: G. H. Wang (gwang@ sio.org.cn)

Received: 23 June 2015 - Published in Ocean Sci. Discuss.: 5 August 2015

Revised: 8 October 2015 - Accepted: 25 October 2015 - Published: 9 November 2015

\begin{abstract}
The sea surface temperature (SST) anomalies in the South China Sea (SCS) and their influences on global atmospheric circulation were studied. The results of a simple atmospheric model suggested that the SCS SST anomalies can induce several barotropic wave trains from the SCS to other regions such as North America, high latitudes of the Southern Hemisphere and the Mediterranean. The baroclinic stream function anomalies from the simple model showed an anticyclonic vortex pair in the Asian continent and the northern and southern Indian Ocean and a cyclonic vortex in the North Pacific and the southwestern Pacific. It is suggested that the spatial pattern of SST anomalies in the SCS can affect the magnitude of stream function anomalies, although it cannot affect the spatial pattern of atmospheric circulation.
\end{abstract}

\section{Introduction}

The South China Sea (SCS, $\left.0-25^{\circ} \mathrm{N}, 100-125^{\circ} \mathrm{E}\right)$ is the largest marginal sea in the northwestern Pacific. The sea surface temperature (SST) in the SCS shows a significant seasonal cycle. The climatological SSTs in summer (JuneAugust) and winter (December-February) over the SCS are shown in Fig. 1. The SST in summer is mostly above $28^{\circ} \mathrm{C}$, with a pronounced cold tongue veering off central Vietnam (Fig. 1a). During winter, the SST is cold in the northwest and warm in the southeast of the SCS (Fig. 1b).

The SST in the SCS had a robust warming trend during the past several decades (Luo et al., 1986; Fang et al., 2006; Xie et al., 2010; Zhang et al., 2010; Liu and Zhang, 2013). Based on the Optimum Interpolation Sea Surface Temperature (OISST) data set, Fang et al. (2006) found that the SST in the SCS had a positive linear trend of $5^{\circ} \mathrm{C} 100 \mathrm{yr}^{-1}$ during 1993-2003. The summer and winter SST trends in the SCS from 1982 to 2011 are also shown in Fig. 1. Whether in summer or in winter, the SCS warming trend is significant, with $1.64^{\circ} \mathrm{C} 100 \mathrm{yr}^{-1}$ in summer and $2.04{ }^{\circ} \mathrm{C} 100 \mathrm{yr}^{-1}$ in winter. The maximum SST trend can exceed $9.50^{\circ} \mathrm{C} 100 \mathrm{yr}^{-1}$. During summer, the larger warming is in the western SCS and the smaller warming in the eastern SCS. During winter, the pattern changes to the larger warming in the eastern SCS and the smaller warming in the western SCS. It should be noted that the SCS warming was faster than the global average, and that the warming was largest between 0 and $20^{\circ} \mathrm{N}$ globally.

Many studies focused on the effects of positive SST anomalies in the SCS on precipitation and climate in China (Zhang et al., 2003; Fong et al., 2004; Roxy and Tanimoto, 2012). According to Zhang et al. (2003), the positive SST anomaly in summer with respect to the seasonal climatology in the SCS was followed by anomalous southward wind and then more moisture was transported to southern China, which resulted in floods in the Yangtze River valley. Fong et al. (2004) suggested that the SCS surface warming can enhance latent and sensible heat fluxes from the sea surface and result in a cyclonic circulation anomaly in the lower troposphere and an anticyclonic circulation anomaly in the upper troposphere, which can then affect the climate of southern China. Roxy and Tanimoto (2012) pointed out that the positive SST anomalies over the SCS tended to form a favorable condition for convective activity and enhanced the northward propagating precipitation anomalies during the SCS summer monsoon. Other studies showed that the SST anomalies in the SCS can influence the SCS monsoon onset (Johnson 


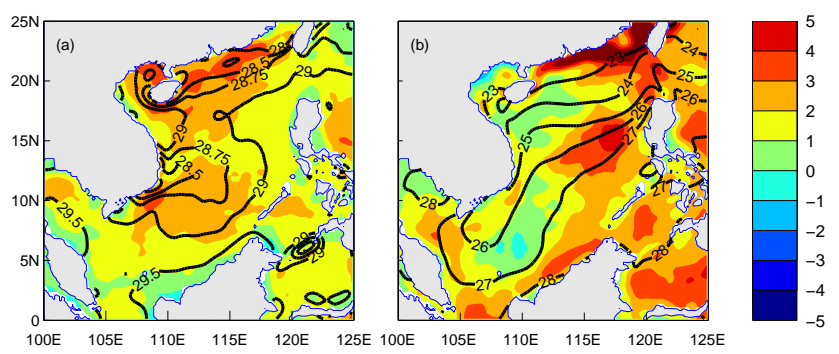

Figure 1. (a) Summer- and (b) winter-mean SST (contours; units: ${ }^{\circ} \mathrm{C}$ ) and SST trends (color shading; units: ${ }^{\circ} \mathrm{C} 100 \mathrm{yr}^{-1}$ ) in the SCS from 1982 to 2011.

and Ciesielski, 2002; Ding et al., 2004; Lestari and Iwasaki, 2006) and its variability (Liu and Xie, 1999; Lestari et al., 2011; Roxy and Tanimoto, 2012).

Teleconnections are well-known and well-studied (Wallace and Gutzler, 1981; Huang, 1984; Nitta, 1986, 1987). A local change in the surface boundary condition can have far reaching influences in a remote area. For example, the diabatic heating anomaly over the central equatorial Pacific during ENSO (El Niño-Southern Oscillation) can excite a stationary barotropic Rossby wave train propagating into extratropical regions. This teleconnection is known as the PacificNorth American (PNA) pattern (Wallace and Gutzler, 1981) in the Northern Hemisphere. Nitta (1987) found another teleconnection between abnormal convective activity over the tropical, western North Pacific and atmospheric circulation anomalies over the mid-latitudes of East Asia in summer, which was named the Pacific-Japan (PJ) pattern.

These studies mostly discussed how the SST in the SCS affected the local climate. The teleconnection between the SCS and global atmosphere circulation is not clear. In this paper, we use a simple atmospheric model to discuss this teleconnection. The rest of this paper is organized as follows. Section 2 describes the data and model used in this study. The results obtained from the simple atmospheric model are presented in Sect. 3. The summary and discussion are provided in Sect. 4.

\section{Data and model}

\subsection{Data and method}

Two data sets are used in this study. The climatological stream functions are from the National Centers for Environmental Prediction/National Center for Atmospheric Research (NCEP/NCAR) reanalysis, which is available on a $2.5^{\circ}$ by $2.5^{\circ}$ grid (Kalnay et al., 1996). The OISST analysis product is from the National Oceanic and Atmospheric Administration (NOAA), which has the spatial resolution of $0.25^{\circ}$ by $0.25^{\circ}$ (Reynolds et al., 2002). The period of the two data sets used is from 1982 to 2011.
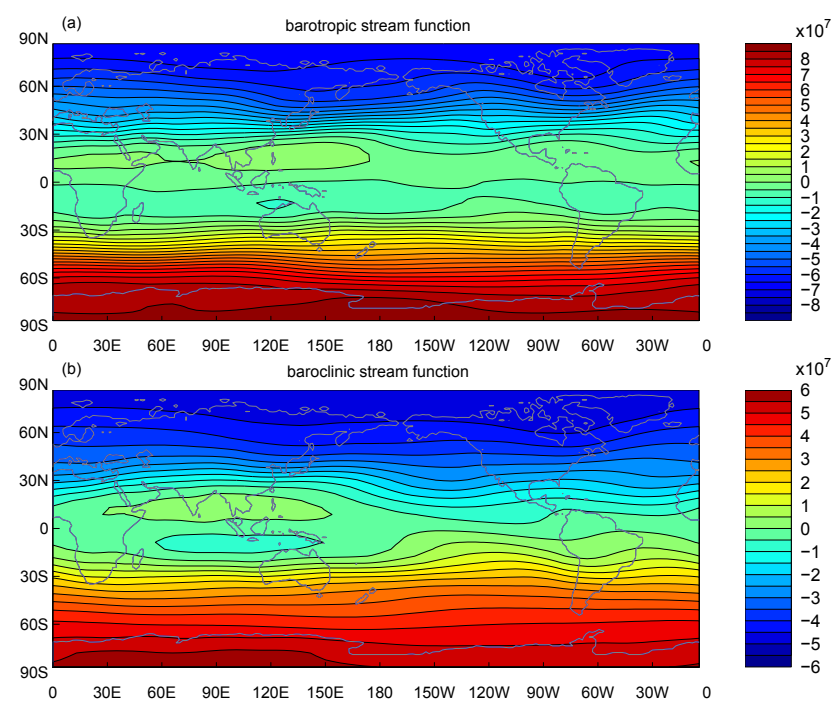

Figure 2. (a) Barotropic and (b) baroclinic stream functions of the climatology. The contour interval is $5.0 \times 10^{6} \mathrm{~m}^{2} \mathrm{~s}^{-1}$.

The basic mean flows are represented by the stream functions at 250 and $750 \mathrm{hPa}$ from the monthly NCEP/NCAR reanalysis. The stream functions at $750 \mathrm{hPa}$ are constructed by linear interpolation from standard pressure levels, as $750 \mathrm{hPa}$ is not a standard pressure level. Since the model atmosphere is simplified to two levels (centered at 250 and $750 \mathrm{hPa}$ ), the stream functions can be separated into barotropic and baroclinic components as follows:

$\psi_{\text {barotropic }}=0.5 \cdot\left(\psi_{250 \mathrm{hPa}}+\psi_{750 \mathrm{hPa}}\right)$,

$\psi_{\text {baroclinic }}=0.5 \cdot\left(\psi_{250 \mathrm{hPa}}-\psi_{750 \mathrm{hPa}}\right)$,

where $\psi$ stands for stream function.

Figure 2 shows the spatial pattern of mean barotropic stream function (Fig. 2a) and baroclinic stream function (Fig. 2b) from 1982 to 2011. Whether barotropic or baroclinic stream functions, it was the westerly in high latitudes. In the tropical regions, the flows fluctuated due to strong convections.

\subsection{Atmospheric model}

We use a simple atmospheric model developed by Lee et al. (2009) to simulate global atmospheric circulation. This is a steady-state, two-level (centered at 250 and $750 \mathrm{hPa}$ ) spherical-coordinate primitive equation model, linearized about prescribed background mean flows. The model uses triangular 18-mode truncations for its horizontal grids. The formulation is similar to that of the multi-level linear baroclinic model used by Hoskins and Simmons (1975) and others, but its governing equations are greatly simplified by employing Gill's (1980) simple thermodynamic equation. A detailed description of the simple model can be found in Lee et al. (2009). This model successfully simulated local and re- 
Table 1. The heat forcing $Q$ at each grid for cases 1-6.

\begin{tabular}{lll}
\hline Exp. & Heat forcing in the SCS & Description \\
\hline Case 1 & $Q(i, j)=Q_{0} *$ & uniform heating \\
Case 2 & $Q(i, j)=Q_{0} \cdot(25-\operatorname{lat}(i, j)) / 12.5$ & heating less in the north \\
Case 3 & $Q(i, j)=Q_{0} \cdot((25-\operatorname{lat}(i, j))+(125-\operatorname{lon}(i, j))) / 25$ & similar to SST winter pattern \\
Case 4 & $Q(i, j)=Q_{0} \cdot\left((12.5-\operatorname{lat}(i, j))^{2} / 10+\operatorname{lon}(i, j)-100\right) / 18.125$ & similar to SST summer pattern \\
Case 5 & $Q(i, j)=Q_{0} \cdot$ summer SST trends pattern $/ 1.64$ & summer SST trends pattern \\
Case 6 & $Q(i, j)=Q_{0} \cdot$ winter SST trends pattern/2.04 & winter SST trends pattern \\
\hline
\end{tabular}

* $Q_{0}=1.16 \times 10^{-2} \mathrm{~W} \mathrm{~kg}^{-1}\left(1^{\circ} \mathrm{Cday}^{-1}\right)$.
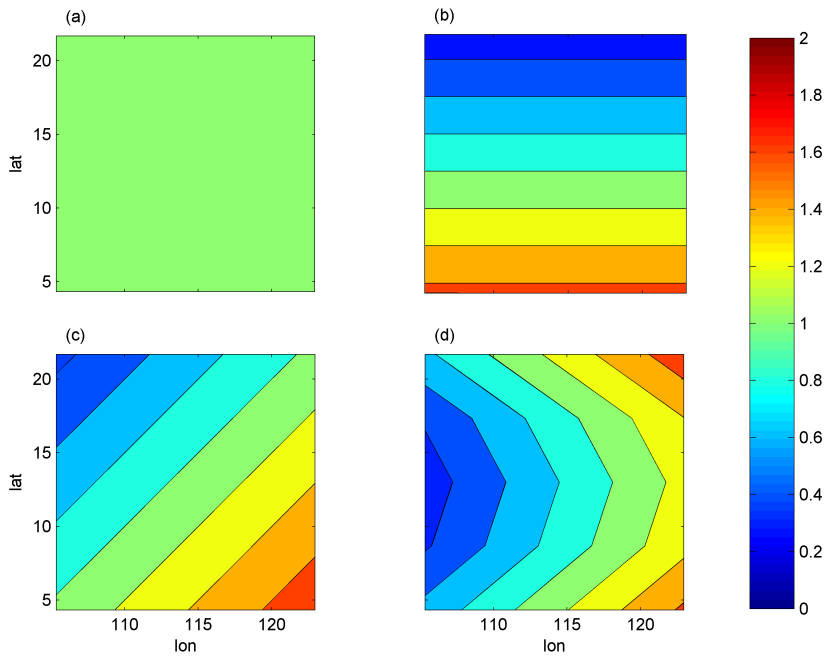

Figure 3. Spatial patterns of heat forcing $Q$ for cases 1-4 (a-d), units: $Q_{0}$.

mote responses of the atmosphere to tropical heating anomalies (Lee et al., 2009; Wang et al., 2010; Zheng et al., 2013). In this study, we use the basic mean flows as the initial conditions and heating in the SCS as the forcing condition to drive this model.

\subsection{Experiment setup}

To see how basin-scale SST anomalies affect the atmospheric circulation, we set six experiments:

- Case 1: uniform heating in the SCS.

- Case 2: heating decreased northward in the SCS, to consider the differences of meridional solar radiation.

- Case 3: heating pattern similar to the SST winter pattern.

- Case 4: heating pattern similar to the SST summer pattern.

- Case 5: summer SST warming trend heating pattern.

- Case 6: winter SST warming trend heating pattern.
Cases 1 and 2 are for testing the effects of the difference of meridional solar radiation. Cases 3 and 4 are for testing the effects of seasonal SST anomalies in the SCS. As shown in Fig. 1, the SST in winter is cooler in the northwestern and warmer in the southeastern SCS, which is used in Case 3, while the pronounced cold tongue veering off central Vietnam in summer is included in Case 4. Heating patterns derived from SST anomalies for these four experiments are summarized in Fig. 3. Cases 5 and 6 are for testing the effects of the SST warming differences in the zonal direction on atmospheric circulation. All calculations are listed in Table 1. Note the total heat input is the same for the six experiments to ensure comparability.

\section{Results}

\subsection{Influence of SST anomalies in the meridional direction}

Figure 4a shows the barotropic stream function anomalies from Case 1. There are three robust waves. The first one is from the SCS to North America through the northwestern Pacific, which is somewhat similar to the classical PNA pattern (Wallace and Gutzler, 1981; Nitta, 1986; Huang, 1984). The second one is from the SCS to high latitudes of the Southern Hemisphere across the Equator. As shown by Wang et al. (2010), the background vertical wind shear is important in converting energy from the heating-induced baroclinic flow anomalies into barotropic motions near the heating source. The barotropic anomalies in turn interact with the mean westerly wind to transmit the barotropic signals to the high latitudes of the Southern Hemisphere. Note another small wave train is from the SCS to the Mediterranean. According to the classical theory of energy dispersion (Yeh, 1949) and the great circle theory (Hoskins and Karoly, 1981), disturbances produced by local heating can spread westward.

The baroclinic stream function anomalies from the simple model show an anticyclonic vortex pair in the Asian continent and the northern and southern Indian Ocean (Fig. 4b). Accordingly, a cyclonic vortex pair appears in the North and southwestern Pacific, quite similar to the Matsuno-Gill model (Gill, 1980) and is consistent with the results of 


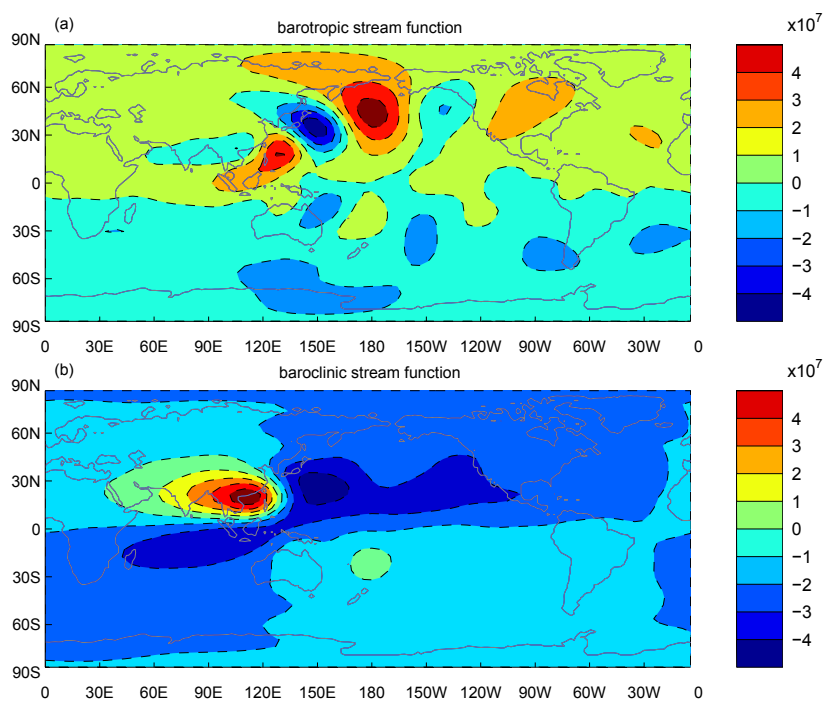

Figure 4. Barotropic (a) and baroclinic (b) stream function anomalies for Case 1 . The contour interval is $1 \times 10^{6} \mathrm{~m}^{2} \mathrm{~s}^{-1}$.
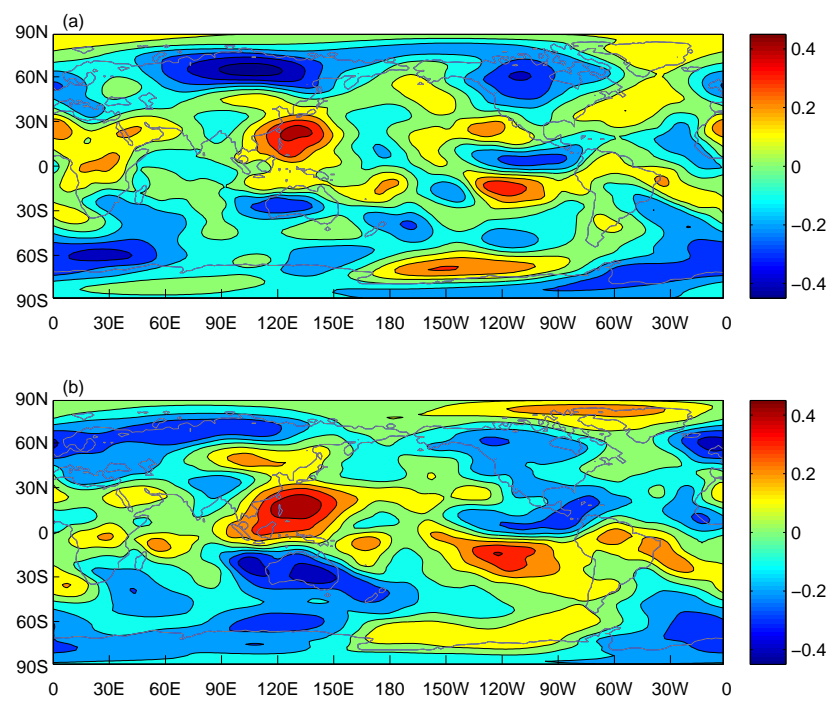

Figure 5. Lag correlation maps (lag day $=15$ days) of the northern SCS $\left(110-121^{\circ} \mathrm{E}, 12-23^{\circ} \mathrm{N}\right) \mathrm{SST}$ anomalies in winter (October 2003-March 2004) with global barotropic stream function (a) and baroclinic stream function (b).

Smagorinsky (1953) and Heckley and Gill (1984). The response of atmospheric circulation to the heating anomaly in the SCS suggests that the Gill dynamics is at work.

We calculate the seasonal cycle of the air-sea temperature difference (figures not shown here). It suggests that atmosphere reduces heat loss to the ocean during the boreal winter in the northern SCS and increases heat flux from the ocean during the summer in the northern SCS. This is supported by $\mathrm{He}$ and $\mathrm{Wu}$ (2013), i.e., that the boreal winter SST in the northern SCS is independent from the atmospheric condition; it gives an opportunity to look at the observational data


Figure 6. (a) Barotropic and (b) baroclinic stream function anomalies (units: $10^{6} \mathrm{~m}^{2} \mathrm{~s}^{-1}$ ) for cases $1-4$, with the black, red, green, and blue contours as stream function anomalies for cases 1,2,3, and 4 , respectively. The contour interval is $2 \times 10^{6} \mathrm{~m}^{2} \mathrm{~s}^{-1}$.

sets and find support for the described teleconnections. The correlations between the winter SST anomalies in the northern SCS and the barotropic/baroclinic stream functions are calculated. The correlation in Fig. 5a shows two waves: one from the SCS to North America and the other from the SCS to the Southern Hemisphere. The correlation in Fig. 5b shows positive anomalies in the Asian continent, northern Indian Ocean and the southwestern Pacific and negative anomalies in the North Pacific and southern Indian Ocean. These support the described teleconnections between SCS SST and the global atmospheric circulation.

The barotropic and baroclinic stream function anomalies for cases 2-4 are basically the same as those for Case 1, which indicates that the positive heating anomalies in the SCS can all induce three waves in the barotropic stream function and two vortex pairs in the baroclinic stream function regardless of the spatial pattern of the heating. The amplitudes are slightly different in the four experiments. For the PNAlike-pattern wave train and the Southern Hemisphere wave train, the barotropic stream functions in cases 1 and 4 are weaker than those in cases 2 and 3 in terms of anticyclonic anomalies, but they are stronger than those in cases 2 and 3 in terms of cyclonic anomalies (Fig. 6a). Conversely, the baroclinic stream functions of cases 1 and 4 are weaker than those in cases 2 and 3 in terms of cyclonic anomalies but are stronger than those in cases 2 and 3 in terms of anticyclonic anomalies (Fig. 6b). The differences among the four cases suggest that the spatial pattern of SST anomalies can affect the magnitudes of both stream functions, although it cannot affect the spatial pattern of the atmospheric circulation. The role of asymmetric heating in influencing atmo- 

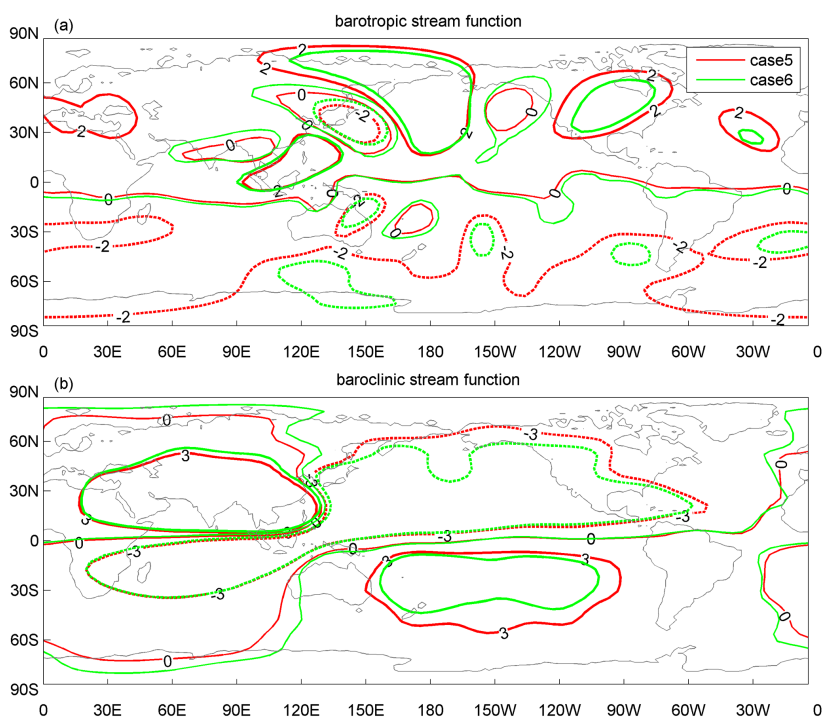

Figure 7. (a) Barotropic and (b) baroclinic stream function anomalies $\left(10^{6} \mathrm{~m}^{2} \mathrm{~s}^{-1}\right)$ for cases 5 (red) and 6 (green). The contour interval is $2 \times 10^{6} \mathrm{~m}^{2} \mathrm{~s}^{-1}$ in the top panel and $3 \times 10^{6} \mathrm{~m}^{2} \mathrm{~s}^{-1}$ in the bottom panel.

spheric circulation can also be seen in many studies such as Fu et al. (1980) and Dunkerton (1989).

\subsection{Influence of SST anomalies in the zonal direction}

The spatial patterns of the stream function anomalies for cases 5 and 6 are also quite similar to those in Case 1 for both barotropic and baroclinic components. For the PNA-likepattern wave train or the Southern Hemisphere wave train, the barotropic stream function in Case 5 is weaker than that in Case 6 in terms of cyclonic anomalies but is stronger than that in Case 6 in terms of anticyclonic anomalies (Fig. 7a). Conversely, the baroclinic stream function in Case 5 is weaker than that in Case 6 in terms of anticyclonic anomalies but is stronger than that in Case 6 in terms of cyclonic anomalies (Fig. 7b). As shown in Fig. 1, the larger warming trend is in the western SCS in summer but in the eastern SCS in winter. The difference between cases 5 and 6 suggests that the larger warming trend in the western (eastern) SCS heating pattern can weaken (strengthen) the cyclonic anomalies and strengthen (weaken) the anticyclonic anomalies in the barotropic component. Conversely, the larger warming trend in the western (eastern) SCS heating pattern can strengthen (weaken) cyclonic anomalies and weaken (strengthen) the anticyclonic anomalies in the baroclinic component. It also suggests that the spatial pattern of the SST trend can affect the magnitude of stream functions, although it cannot affect the spatial pattern of atmospheric circulation.

\section{Summary and discussion}

In this study, the influences of SST anomalies in the SCS on global atmospheric circulation were studied. The results of the simple atmospheric model suggested that the SCS heating can induce a barotropic wave train from the SCS to the northwestern Pacific Ocean and North America, which is somewhat similar to the classical PNA pattern. Simultaneously, the SCS heating can induce a barotropic wave train from the SCS to high latitudes of the Southern Hemisphere. In particular, we noticed a weak barotropic wave train from the western SCS to the Mediterranean. The baroclinic stream function anomalies from the simple model showed an anticyclonic vortex pair in the Asian continent and the northern and southern Indian Ocean and a cyclonic vortex in the North Pacific and the southwestern Pacific. The stream function anomalies of the barotropic and baroclinic components for all six cases are basically the same, with slight differences in amplitude. This suggests that the spatial pattern of heating can cause some differences in magnitude but not in circulation patterns.

Our findings in this study may be important for both regional and global climate research. For example, we calculated the correlation between the northern SCS SST anomalies and the rainfall. The correlation pattern is quite similar to Fig. 4a, showing two waves: one from the SCS to North America and the other from the SCS to the Southern Hemisphere (figures not shown here); thus, this study may help to forecast climate-related events like rainfall in North America based on the SCS SST anomalies.

Because the two-level model applied here only considers Gill's (1980) simple thermodynamics equation, many dynamics/thermodynamics are ignored completely. Thus, a more complex atmospheric general circulation model is needed for further study.

Acknowledgements. The authors thank Sang-Ki Lee and Chunzai Wang for sharing the model code. The authors are grateful to the two anonymous reviewers who have helped in improving the manuscript considerably. This study was supported by the National Basic Research Program of China (grant no. 2012CB955601) and the National Natural Science Foundation of China (grant no. 41125019 and 91428206).

Edited by: M. Meier

\section{References}

Ding, Y. H., Li, C. Y., and Liu, Y. J.: Overview of the South China Sea monsoon experiment, Adv. Atmos. Sci., 21, 343-360, doi:10.1007/BF02915563, 2004.

Dunkerton, T. J.: Nonlinear Hadley circulation driven by asymmetric differential heating, J. Atmos. Sci., 46, 956-974, 1989.

Fang, G., Wei, H., Chen, Z., Wang, Y., Wang, X., and Li, C.: Trends and interannual variability of the South China Sea surface winds, 
surface height, and surface temperature in the recent decade, J. Geophys. Res., 111, C11S16, doi:10.1029/2005JC003276, 2006.

Fong, S. K., Wu, C. S., Wang, A. Y., Ku, C. M., Hao, I. P., and Tong, T. N.: A numerical study of the effects of South China Sea SST anomalies on the climate in South China, J. Trop. Meteorol., 20, 32-38, 2004 (in Chinese).

Fu, K. Z., Wu, H. D., Fang, X. F., and Wang, Y. K.: Asymmetric heating effects on the general circulation of atmosphere, Acta Meteorol. Sin., 38, 205-218, 1980 (in Chinese).

Gill, A. E.: Some simple solutions for heat-induced tropical circulation, Q. J. Roy. Meteorol. Soc., 106, 447-462, doi:10.1002/qj.49710644905, 1980.

He, Z. Q. and Wu, R. G.: Seasonality of interannual atmosphereocean interaction in the South China Sea, J. Oceanogr., 69, 699712, doi:10.1007/s10872-013-0201-9, 2013.

Heckley, W. A. and Gill, A. E.: Some simple analytical solutions to the problem of forced equatorial long waves, Q. J. Roy. Meteorol. Soc., 110, 203-217, doi:10.1002/qj.49711046314, 1984

Hoskins, B. J. and Karoly, D. J.: The steady linear response of a spherical atmosphere to thermal and orographic forcing, J. Atmos. Sci., 38, 1179-1196, doi:10.1175/15200469(1981)038<1179:TSLROA>2.0.CO;2, 1981.

Hoskins, B. J. and Simmons, A. J.: A multi-layer spectral model and the semi-implicit method, Q. J. Roy. Meteorol. Soc., 101, 637-655, doi:10.1002/qj.49710142918, 1975.

Huang, R. H.: The characteristics of the forced stationary planetary wave propagations in summer Northern Hemisphere, Adv. Atmos. Sci., 1, 84-94, doi:10.1007/BF03187619, 1984.

Johnson, R. H. and Ciesielski, P. E.: Characteristics of the 1998 summer monsoon onset over the northern South China Sea, J. Meteorol. Soc. Jpn. Ser. II, 80, 561-578, doi:10.2151/jmsj.80.561, 2002.

Kalnay, E., Kanamitsu, M., Kistler, R., Collins, W., Deaven, D., Gandin, L., Iredell, M., Saha, S., White, G., Woollen, J., Zhu, Y., Leetmaa, A., Reynolds, B., Chelliah, M., Ebisuzaki, W., Higgins, W., Janowiak, J., Mo, K. C., Ropelewski, C., Wang, J., Jenne, R., and Joseph, D.: The NCEP/NCAR 40-year reanalysis project, B. Am. Meteorol. Soc., 77, 437-471, doi:10.1175/15200477(1996)077<0437:TNYRP>2.0.CO;2, 1996.

Lee, S. K., Wang, C. Z., and Mapes, B. E.: A simple atmospheric model of the local and teleconnection responses to tropical heating anomalies, J. Climate, 22, 272-284, doi:10.1175/2008JCLI2303.1, 2009.

Lestari, R. K. and Iwasaki, T.: A GCM study on the roles of the seasonal marches of the SST and land-sea thermal contrast in the onset of the Asian summer monsoon, J. Meteorol. Soc. Jpn. Ser. II, 84, 69-83, 2006.

Lestari, R. K., Watanabe, M., and Kimoto, M.: Role of air-sea coupling in the interannual variability of the South China Sea summer monsoon, J. Meteorol. Soc. Jpn. Ser. II, 89, 283-290, doi:10.2151/jmsj.2011-A18, 2011.

Liu, Q. and Zhang, Q.: Analysis on long-term change of sea surface temperature in the China Seas, J. Ocean Univ. China, 12, 295300, doi:10.1007/s11802-013-2172-2, 2013.
Liu, W. T. and Xie, X.: Space-based observations of the seasonal changes of South Asian monsoons and oceanic response, Geophys. Res. Lett., 26, 1473-1476, doi:10.1029/2003JC001867, 1999.

Luo, S. H. and Jin, Z. H.: Statistical analyses for sea surface temperature over the South China Sea, behavior of subtropical high over the west pacific and monthly mean rainfall over the Chang Jiang middle and lower reaches, J. Atmos. Sci., 10, 409-418, 1986 (in Chinese).

Nitta, T.: Long-term variations of cloud amount in the western Pacific region, J. Meteorol. Soc. Jpn., 64, 373-390, 1986.

Nitta, T.: Convective activities in the tropical western pacific and their impact on the northern hemisphere circulation, J. Meteorol. Soc. Jpn., 65, 373-390, 1987.

Reynolds, R. W., Rayner, N. A., Smith, T. M., Stokes, D. C., and Wang, W. Q.: An improved in situ and satellite SST analysis for climate, J. Climate, 15, 1609-1625, doi:10.1175/15200442(2002)015<1609:AIISAS>2.0.CO;2, 2002.

Roxy, M. and Tanimoto, Y.: Influence of sea surface temperature on the intraseasonal variability of the South China Sea summer monsoon, Clim. Dynam., 39, 1209-1218, doi:10.1007/s00382011-1118-x, 2012.

Smagorinsky, J.: The dynamical influence of large-scale heat sources and sinks on the quasi-stationary mean motions of the atmosphere, Q. J. Roy. Meteor. Soc., 79, 342-366, doi:10.1002/qj.49707934103, 1953.

Wallace, J. M. and Gutzler, D. S.: Teleconnections in the geopotential height field during the Northern Hemisphere winter, Mon. Weather Rev., 109, 784-812, doi:10.1175/15200493(1981)109<0784:TITGHF>2.0.CO;2, 1981.

Wang, C. Z., Lee, S. K., and Mechoso, C. R.: Interhemispheric influence of the Atlantic warm pool on the southeastern Pacific, J. Climate, 23, 404-418, doi:10.1175/2009JCLI3127.1, 2010.

Xie, S. P., Deser, C., Vecchi, A. G., Ma, J., Tend, H., and Wittenberg, A. T.: Global warming pattern formation: sea surface temperature and rainfall, J. Climate, 23, 966-986, doi:10.1175/2009JCLI3329.1, 2010.

Yeh, T.: On energy dispersion in the atmosphere, J. Meteorol., 6, 1-16, doi:10.1175/15200469(1949)006<0001:OEDITA>2.0.CO;2, 1949.

Zhang, L. P., Wu, L. X., Lin, X. P., and Wu, D. X.: Modes and mechanisms of sea surface temperature low-frequency variations over the coastal China seas, J. Geophys. Res., 115, C08031, doi:10.1029/2009JC006025, 2010.

Zhang, Q., Liu, P., and Wu, G. X.: The relationship between the flood and drought over the lower reach of the Yangtze River Valley and the SST over the Indian Ocean and the South China Sea, J. Atmos. Sci., 27, 992-1006, 2003 (in Chinese).

Zheng, J., Liu, Q. Y., Wang, C. Z., and Zheng, X. T.: Impact of heating anomalies associated with rainfall variations over the indowestern Pacific on Asian atmospheric circulation in winter, Clim. Dynam., 40, 2023-2033, doi:10.1007/s00382-012-1478-x, 2013. 